\title{
Diversité et gestion génétique de la truite commune : apport de l'étude du polymorphisme des locus protéiques et microsatellites
}

\author{
P Presa, F Krieg, A Estoup, R Guyomard \\ Institut national de la recherche agronomique, \\ laboratoire de génétique des poissons, 78352 Jouy-en-Josas Cedex, France
}

Résumé - Nous avons examiné le polymorphisme électrophorétique de 47 locus enzymatiques dans 24 populations naturelles et domestiques de truite commune de France ainsi que la variabilité à 5 locus microsatellites dans 4 de ces populations. La proportion de locus enzymatiques polymorphes sur l'ensemble des échantillons est de $57 \%$ et varie de 0 à $37 \%$ entre populations. Ce polymorphisme permet de distinguer 2 sous-espèces distinctes occupant respectivement les bassins atlantique et méditerranéen et dont la distance génétique standard (Nei) s'établit, en moyenne, à 0,10 . La variabilité des populations atlantiques s'avère nettement plus élevée que celle des populations méditerranéennes et présente un certain degré de structuration géographique. Toutes les souches domestiques étudiées ont une origine atlantique. Les 5 locus microsatellites examinés se sont avérés polymorphes avec un nombre d'allèles variant de 2 à 8 et des taux d'hétérozygotie intrapopulation de 0 à $74 \%$. Ils permettent aussi de distinguer la population méditerranéenne étudiée des 3 pópulations d'origine atlantique. L'intérêt de ces résultats pour la gestion de la diversité génétique des populations naturelles de truite commune est brièvement présenté.

protéine / microsatellite / polymorphisme moléculaire / gestion génétique / Salmo trutta

Summary - Genetic diversity and management of brown trout: the value of studying variation at protein and microsatellite loci. The electrophoretic polymorphism at 47 protein loci was studied in 24 domesticated and natural French brown trout populations. The variation at 5 microsatellite loci in 4 of these populations was also investigated. The total proportion of polymorphic loci was $57 \%$ for all the populations analysed and ranged from 0 to $37 \%$ between populations. This polymorphism allowed us to distinguish 2 subspecies which occupy the Atlantic and Mediterranean areas, respectively. The average standard genetic distance between these 2 groups was estimated to be 0.10 . The genetic diversity of the Atlantic populations was substantially higher than that of the Mediterranean populations and showed some geographic structuration. All the domesticated stocks originated from the Atlantic area. The 5 microsatellite loci were polymorphic with a total number of alleles per locus ranging from 2 to 8 and heterozygosity was between 0 and $74 \%$. These microsatellite loci also allowed us to distinguish the Mediterranean population from the 3 populations 
originating from the Atlantic area. The value of these results for the genetic management of brown trout is briefly discussed.

protein / microsatellite / molecular polymorphism / genetic management / Salmo trutta

\section{INTRODUCTION}

Les principales espèces de poissons dulçaquicoles d'intérêt économique sont constituées à la fois de populations naturelles exploitées par la pêche et de souches domestiques destinées à la production commerciale ou au repeuplement.

La constitution des stocks domestiques a été réalisée sans tenir compte de la diversité intraspécifique, généralement mal connue. Cette. attitude peut avoir 2 conséquences négatives : 1) une domestication qui n'exploite qu'une faible part des potentialités génétiques de l'espèce; 2 ) une réduction de la diversité génétique et du potentiel adaptatif des populations naturelles par introgression répétée de celles-ci avec des souches de repeuplement d'origine non identifiée. Ces problèmes se posent avec d'autant plus d'acuité que ces espèces présentent :

- une fécondité généralement très élevée qui autorise à la fois des effets fondateurs très marqués (entretien de souches domestiques avec peu de géniteurs) et des explosions démographiques (propagation en milieu naturel de sujets issus d'un nombre réduit de souches domestiques, voire d'une seule);

- une diversité interpopulation souvent très importante, à relier au fait que leur domaine est fragmenté en bassins hydrographiques indépendants.

Une stratégie rationnelle de gestion des ressources génétiques des espèces «semidomestiquées» de poissons reste à promouvoir en s'appuyant sur 3 volets : 1) une description précise de la diversité génétique de ces espèces; 2) la fondation de nouveaux stocks captifs reproduits en pisciculture et destinés soit au repeuplement, soit à la production commerciale ; 3 ) la constitution de conservatoires de gènes.

La truite commune (Salmo trutta L) constitue un cas exemplaire de poisson d'eau douce semi-domestiqué. Elle possède une aire naturelle de distribution très vaste (Europe, Anatolie et Atlas) et présente une forte diversité phénotypique qui a conduit à distinguer de nombreuses formes géographiques et écologiques. Cette espèce, qui symbolise la qualité d'un environnement, est exploitée par pêche sportive et professionnelle sur tout son domaine. À ce titre, elle fait l'objet de repeuplements intensifs (représentant environ $80 \%$ des déversements réalisés dans les rivières ou plans d'eau français) à partir de quelques souches domestiques. Son potentiel élevé pour la survie et croissance en mer en fait aussi une candidate intéressante pour l'aquaculture marine (Krieg et al, 1992) et l'évaluation des possibilités d'amélioration génétique de la croissance a abouti à des résultats très encourageants (Chevassus et al, 1992). La truite commune apparaît donc comme un modèle privilégié pour entreprendre une réflexion sur la gestion des ressources génétiques d'une espèce de poisson dulçaquicole semi-domestiquée et tester un certain nombre de solutions. En outre, sa structure fortement polytypique et l'existence de zones d'hybridation provoquées par le repeuplement en font 
un organisme particulièrement favorable à l'étude des caractères et mécanismes impliqués dans la différenciation et l'adaptation chez un poisson d'eau douce.

Pour décrire précisément la structure génétique de cette espèce, 3 méthodes permettant respectivement de mettre en évidence le polymorphisme des locus protéiques, des locus microsatellites et de portions de séquences de l'ADN mitochondrial ont été développées. Nous présentons ici les résultats majeurs obtenus avec les 2 premières méthodes dans le cas des populations françaises et en discutons l'intérêt pour la gestion génétique de l'espèce.

\section{MATÉRIEL ET MÉTHODES}

La figure 1 représente la localisation géographique des populations naturelles de truite commune dont le polymorphisme enzymatique est décrit dans cet article, le nombre d'individus par échantillon variant de 15 à 30 selon les sites. Ces sites correspondent à des secteurs pas ou peu repeuplés. Les échantillons de souches domestiques ont été choisis sur une base géographique assez large (France, Italie, Danemark) dans les piscicultures de repeuplement les plus importantes.

Les souches domestiques analysées sont : Etrun (Etru), pisciculture du Pas-deCalais; Vermenoux (Verm), pisciculture de l'Yonne; Gournay (Gour), pisciculture expérimentale INRA; Roquebillière (Roqu), pisciculture des Alpes maritimes; La Canourgue (Cano), pisciculture de la Lozère; Cuneo (Cuné), pisciculture du Piémont italien; Cracovie (Crac), pisciculture polonaise des Tatras.

Sur chaque individu, ont été prélevés un œil, du muscle, du foie et du sang. Ces prélèvements ont été immédiatement congelés dans l'azote liquide ou la glace carbonique. Un aliquot de sang a été dissous dans du TNES-Urée 4M pour l'extraction d'ADN nucléaire. Les techniques d'électrophorèse de protéines, de clonage et d'électrophorèse de microsatellites sont respectivement décrites dans Krieg (1984), Krieg et Guyomard (1985) et Estoup (1992). La nomenclature des systèmes, locus et allèles est conforme aux recommandations de Shaklee et al (1990). Lorsque cela était possible, nous avons retenu, pour des allèles identiques, les codes proposés par d'autres auteurs. La taille exacte des allèles observés aux différents locus microsatellites a été estimée par comparaison avec une séquence de référence. Un dendrogramme des populations naturelles et domestiques de truite commune a été obtenu par agglomération hiérarchique de type UPGMA à partir d'une matrice de distances génétiques standard calculées selon Nei (1975). La variabilité intrapopulation (taux d'hétérozygotie, H) a été estimée par la diversité génique intrapopulation (Nei, 1975). Le PIC (polymorphism information content) à un locus a été estimé par : $\Sigma\left(1-p_{i}^{2}\right)-\Sigma\left(p_{i} p_{j}\right)^{2}$ où $p_{i}$ et $p_{j}$ représentent les fréquences des allèles $i$ et $j$ (Botstein et al, 1980). 


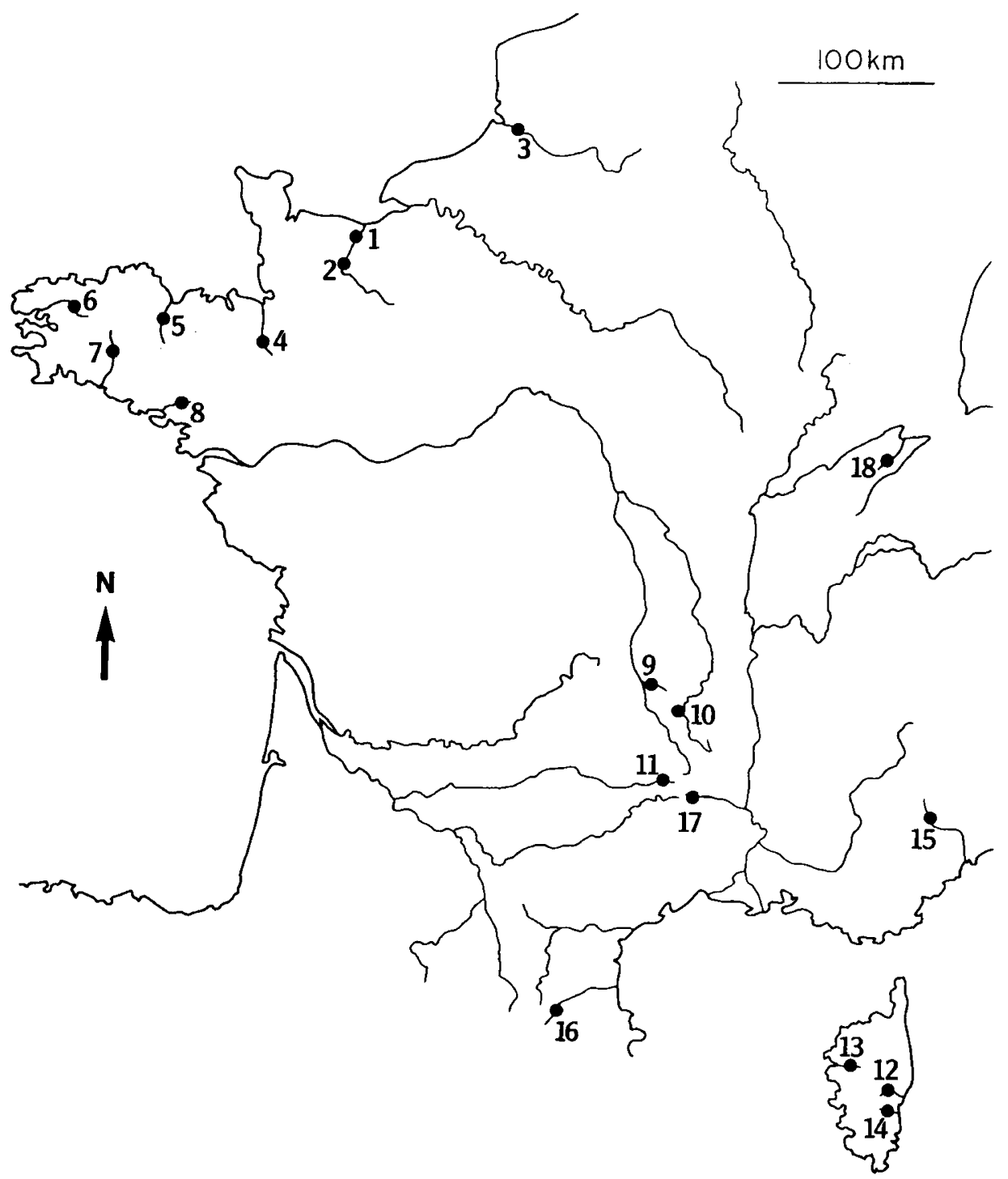

Fig 1. Localisation géographique des populations naturelles étudiées. 1) Orne, phénotype truite de mer (TmeO) ; 2) Orne, phénotype sédentaire (SedO) ; 3) Bresle, phénotype truite de mer (TmeB) ; 4) Avion (Avio) ; 5) Leffe (Leff) ; 6) Elorn (Elor); 7) Kernec, affluent du Scorff (Kern) ; 8) Montgolérian (Mont) ; 9) Allier (Alli) ; 10) Loire (Loir) ; 11) Lot (Lot); 12) Travo (Trav); 13) Aitone (Aito); 14) Solenzara (Sole); 15) Coulomp, affluent du Var (Coul) ; 16) Maureillas, affluent du Tech (Maur); 17) Luech, affluent de la Cèze (Luec); 18) Reverotte : bassin du Doubs (Reve). 


\section{RÉSULTATS}

\section{Variabilité des locus enzymatiques}

Le tableau I énumère l'ensemble des 20 systèmes et 47 locus analysés en routine dans les populations. Les patrons électrophorétiques observés ont été décrits antérieurement (Krieg, 1984). Les déterminismes génétiques proposés pour expliquer les variations observées ont été vérifiés, pour la plupart, par des croisements de contrôle (Guyomard et Krieg, 1983; Barbat-Leterrier et al, 1989).

La proportion de locus polymorphes, calculée sur l'ensemble des populations est de $57 \%$ (27 sur 47 ) et varie de $0 \%$ (Travo) à $37 \%$ (Leffe). Le tableau II présente les fréquences alléliques observées aux 13 locus protéiques les plus polymorphes.

L'examen détaillé de ce tableau fait clairement apparaître une dichotomie entre, d'une part, les populations naturelles atlantiques et, d'autre part, les populations naturelles méditerranéennes. Les différences sont particulièrement marquantes aux locus $F B P-1^{*}, F H-1,2^{*}, L D H-5^{*}, M P I-2^{*}$ et $T F^{*}$. Si l'on met à part la population du Travo (Corse), le dendrogramme (fig 2) met aussi clairement en évidence la même dichotomie. La forte divergence apparente de la population du Travo ne correspond pas nécessairement à la présence de 2 entités génétiques distinctes dans les bassins hydrographiques corses, mais résulte plus vraisemblablement d'une forte dérive génétique liée à un "goulot d'étranglement» (absence totale d'hétérozygotes et fixation à 5 locus d'allèles présent dans d'autres populations corses, mais à des fréquences faibles).

Les populations méditerranéennes se distinguent aussi des populations atlantiques par des niveaux moyens d'hétérozygotie plus faibles : ceux-ci varient de 1,8 à $10,9 \%$ (moyenne $=6,59$ ) dans les populations atlantiques et de 0 à $4,7 \%$ (moyenne $=2,05$ ) dans les populations méditerranéennes (tableau II). La distance génétique standard moyenne entre les 2 principaux groupes atteint 0,10 environ. Les populations atlantiques constituent le groupe le plus variable avec une variabilité totale égale à 0,10 (fig 3); $40 \%$ de cette variabilité est due aux différences entre populations (fig 3 ) et une tendance à des regroupements géographiques se dessine; c'est le cas, par exemple, des échantillons de la pointe de la Bretagne (sites 6 et 7; fig 1 et 2) qui se caractérisent par la présence de $F H-1,2^{*} 75, S O D-1^{*} 80$ et $L D H$ $5^{*} 100$. Les populations corses présentent aussi une variabilité totale presque aussi élevée que les populations atlantiques $(0,08$; fig 3$)$. En comparaison, les populations méditerranéennes continentales forment un ensemble plus homogène (variabilité totale $=0,04)$. Enfin, si l'on considère l'ensemble des populations naturelles étudiées, la variabilité inter-population représente un peu plus de la moitié de la variabilité génétique totale (fig 3).

Les populations domestiques examinées se rattachent sans ambiguïté au groupe atlantique. Elles sont très peu différenciées les unes des autres et présentent un niveau d'hétérozygotie élevé (fig 3 ). Il est important de noter que ces souches sont toutes fixées pour les allèles $L D H-5^{*} 90$ et $T F^{*} 100$ tandis que les populations méditerranéennes étudiées le sont pour les allèles $L D H-5^{*} 100$ et $T F^{*} 102 . L D H-5^{*}$ et $T F^{*}$ constituent donc 2 marqueurs génétiques pour suivre les effets du repeuplement dans les rivières méditerranéennes. Quatre autres locus $\left(F B P-1^{*}, F H-1,2^{*}\right.$, $\left.M P I-2^{*}\right)$ auxquels les souches domestiques possèdent des allèles absents des populations méditerranéennes peuvent aussi permettre de confirmer des phénomènes 
Tableau I. Déterminisme génétique des systèmes enzymatiques examinés chez la truite commune.

\begin{tabular}{|c|c|c|c|}
\hline Système enzymatique & Tissu & Structure & Locus \\
\hline Aspartate aminotransférase (AAT) & $\begin{array}{l}\text { Muscle } \\
\text { Foie }\end{array}$ & $\begin{array}{l}\text { Dimère } \\
\text { Dimère }\end{array}$ & $\frac{A A T-1}{A A T-4}^{*}$ et $\underline{2}^{*}$ \\
\hline Alcool déshydrogénase (ADH) & Foie & Dimère & $A D H^{*}$ \\
\hline Glycérol-3-phosphate Déshydrogénase (G3PDH) & Muscle & Dimère & ${\underline{G 3 P D H-2^{*}}}^{*}$ \\
\hline Adénylate kinase $(\mathrm{AK})$ & Muscle & Monomère & $A K^{*}$ \\
\hline Créatine kinase (CK) & $\begin{array}{l}\text { Muscle } \\
\text { (Eil }\end{array}$ & $\begin{array}{l}\text { Dimère } \\
\text { Dimère }\end{array}$ & $\frac{C K-1^{*}}{C K-3^{*}}$ et $\frac{2^{*}}{4^{*}}$ \\
\hline Estérase (EST) & $\begin{array}{l}\text { Foie } \\
\text { Sérum } \\
\text { Muscle }\end{array}$ & $\begin{array}{l}\text { Monomère } \\
\text { Monomère } \\
\text { Monomère }\end{array}$ & $\begin{array}{l}E S T-1^{*} \\
E S T-2^{*} \\
E S T-5^{*}\end{array}$ \\
\hline Fructose biphosphate (FDP) & Foie & Dimère & $E B P-1^{*}$ et $\underline{2}^{*}$ \\
\hline Fumarase hydratase (FUM) & Muscle & Tétramère & ${\underline{F H}-1^{*}}^{*} \underline{2}^{*}$ \\
\hline Isocitrate Déhydrogénase (IDHP) & $\begin{array}{l}\text { Muscle } \\
\text { Foie }\end{array}$ & $\begin{array}{l}\text { Dimère } \\
\text { Dimère }\end{array}$ & $\begin{array}{l}I D H P-1^{*} \text { et } 2^{*} \\
I D H P-3^{*} \text { et } 4^{*}\end{array}$ \\
\hline Lactate déshydrogénase (LDH) & $\begin{array}{l}\text { Muscle } \\
\text { Foie } \\
\text { CEil }\end{array}$ & $\begin{array}{l}\text { Tétramère } \\
\text { Tétramère } \\
\text { Tétramère }\end{array}$ & $\begin{array}{l}L D H-1^{*} \text { et } \mathscr{2}^{*} \\
\frac{L D H-3^{*}}{L D H-5^{*}} \text { et } \underline{4}^{*}\end{array}$ \\
\hline Malate déhydrogénase (MDH) & $\begin{array}{l}\text { Foie } \\
\text { Muscle }\end{array}$ & $\begin{array}{l}\text { Dimère } \\
\text { Dimère }\end{array}$ & ${\underline{M D H-1^{*}}}^{*}$ et $\underline{2}^{*}$ \\
\hline Enzyme malique (MEP) & $\begin{array}{l}\text { Muscle } \\
\text { Foie }\end{array}$ & $\begin{array}{l}\text { Tétramère } \\
\text { Tétramère }\end{array}$ & $\begin{array}{l}M E P-1^{*} \text { et } 2^{*} \\
M E P-3^{*} \text { et } 4^{*}\end{array}$ \\
\hline Para-albumine (PALB) & Sérum & Monomère & $P A L B-1^{*}$ et $\mathscr{2}^{*}$ \\
\hline Phosphogluconate déshydrogénase (PGDH) & Muscle & Dimère & ${\underline{P G D H-2^{*}}}^{*}$ \\
\hline Phosphoglucomutase (PGM) & Muscle & Monomère & $P G M-2^{*}$ \\
\hline Glucose-6-phosphate isomérase (GPI) & $\begin{array}{l}\text { Muscle } \\
\text { CEil }\end{array}$ & $\begin{array}{l}\text { Dimère } \\
\text { Dimère }\end{array}$ & $\frac{G P I-1^{*}}{G P I-3^{*}}$ et $\underline{2}^{*}$ \\
\hline Phosphomannose isomérase (PMI) & Muscle & Monomère & $\underline{M P I-2^{*}}$ \\
\hline L-Iditol déshydrogénase (IDDH) & Foie & Tétramère & $\underline{I D D H-1^{*}}$ et $\underline{2}^{*}$ \\
\hline Superoxyde dismutase (SOD) & $\begin{array}{l}\text { Foie } \\
\text { Foie }\end{array}$ & $\begin{array}{l}\text { Tétramère } \\
\text { Dimère }\end{array}$ & $\begin{array}{l}S O D-1^{*} \\
S O D-2^{*}\end{array}$ \\
\hline Transferrine (TF) & Sérum & Monomère & $T F^{*}$ \\
\hline
\end{tabular}

Les locus analysés en croisements de contrôle sont soulignés.

d'introgression consécutifs au repeuplement. Les fréquences observées à ces 6 locus permettent de vérifier, a posteriori, que les échantillons méditerranéens ont été effectivement prélevés sur des sites non contaminés par le repeuplement. 


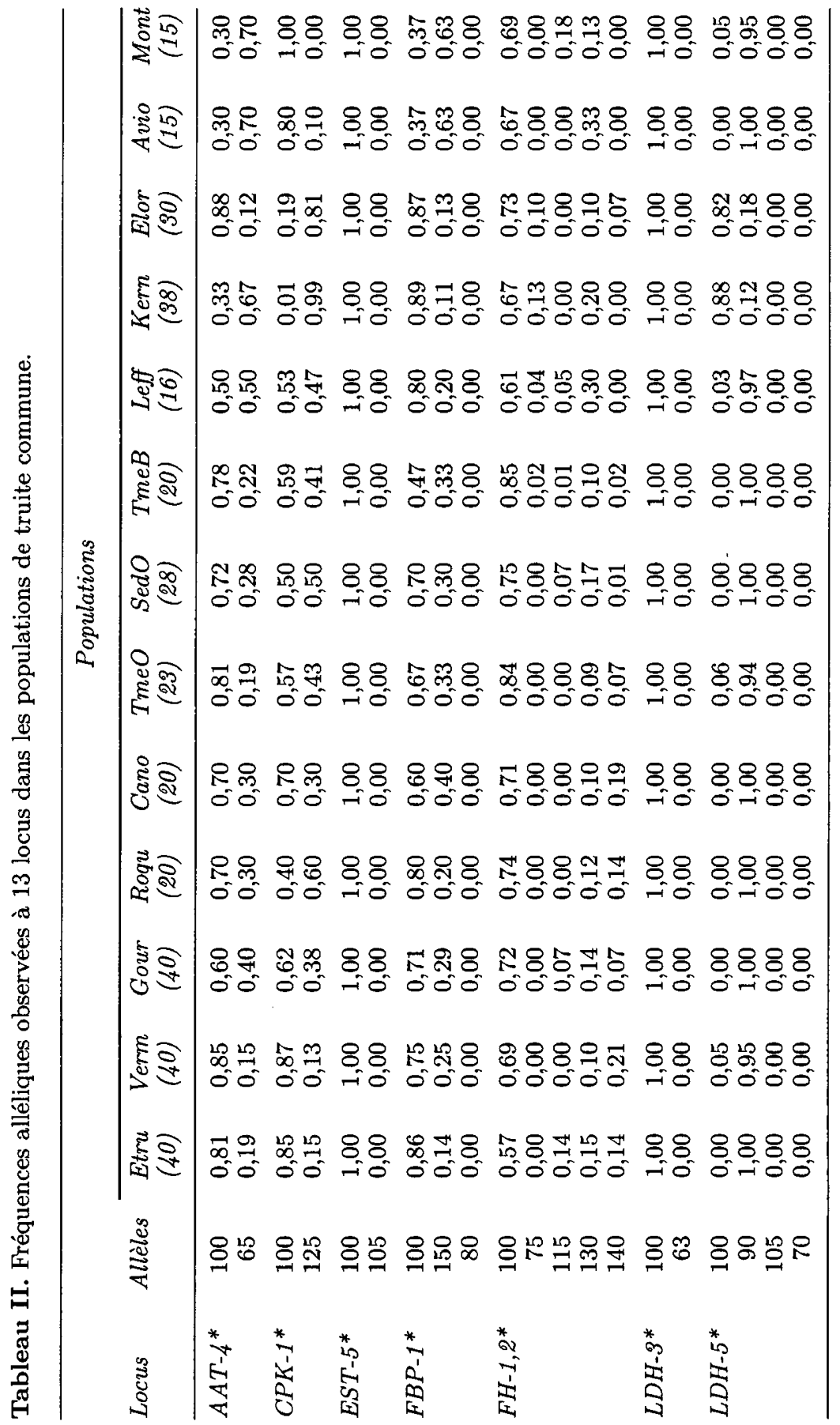




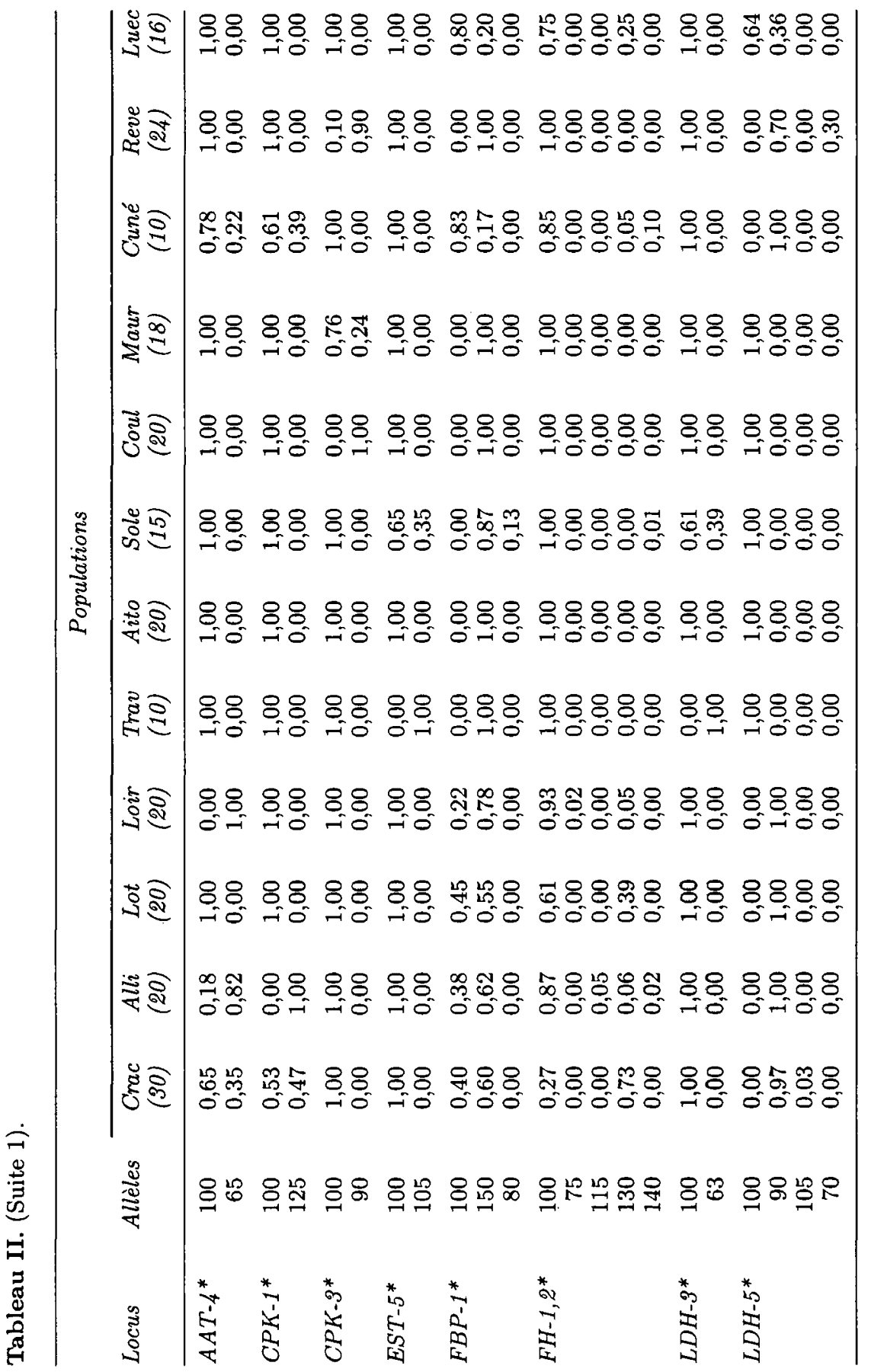




\begin{tabular}{|c|c|c|}
\hline 2 & 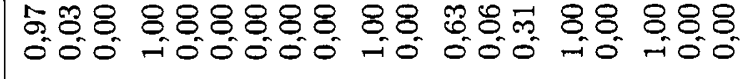 & $\underset{*}{F}$ \\
\hline$\frac{\cos }{2}$ & 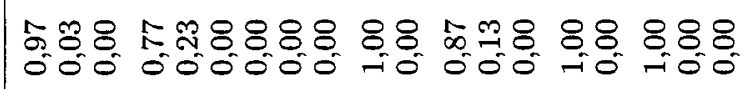 & $\stackrel{\infty}{\circ}$ \\
\hline 蟋 & 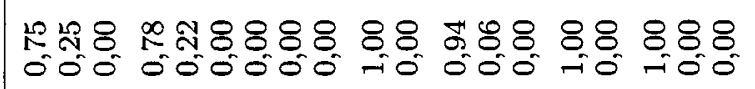 & $\begin{array}{l}\overrightarrow{0} \\
i 0^{5}\end{array}$ \\
\hline 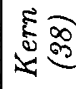 & 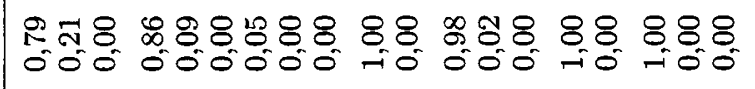 & 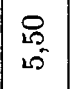 \\
\hline & 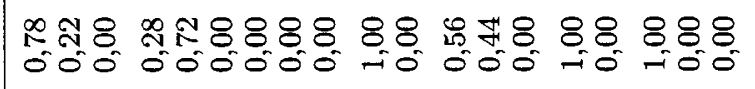 & 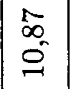 \\
\hline हैำ & 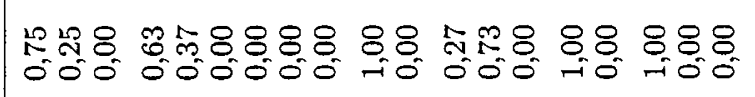 & $\begin{array}{l}\mathscr{8} \\
\infty \\
\infty\end{array}$ \\
\hline : & 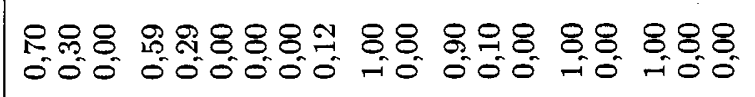 & $\ddot{G}$ \\
\hline & 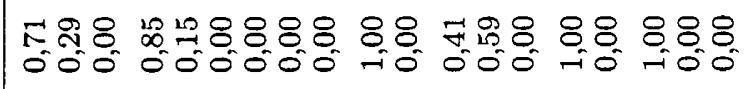 & 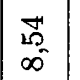 \\
\hline & 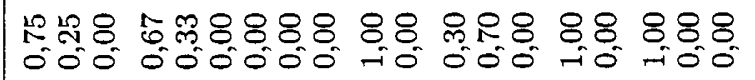 & $\begin{array}{c}8 \\
\infty \\
\infty\end{array}$ \\
\hline & 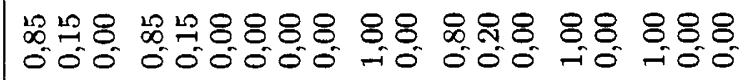 & $\underset{\substack{\mathrm{S} \\
\mathrm{N}}}{2}$ \\
\hline & 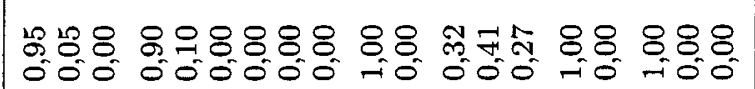 & $\frac{\pi}{s}$ \\
\hline 동 & 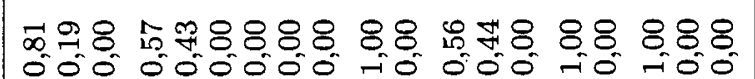 & $\frac{9}{\infty}$ \\
\hline & 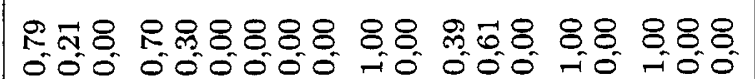 & 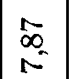 \\
\hline 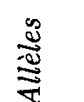 & 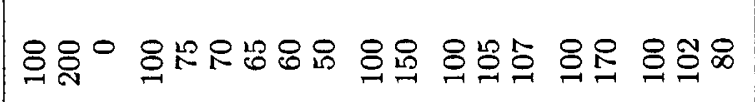 & \\
\hline 胥 & 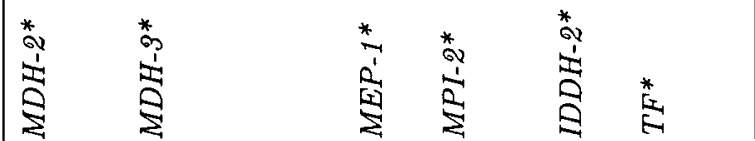 & 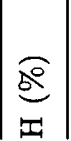 \\
\hline
\end{tabular}




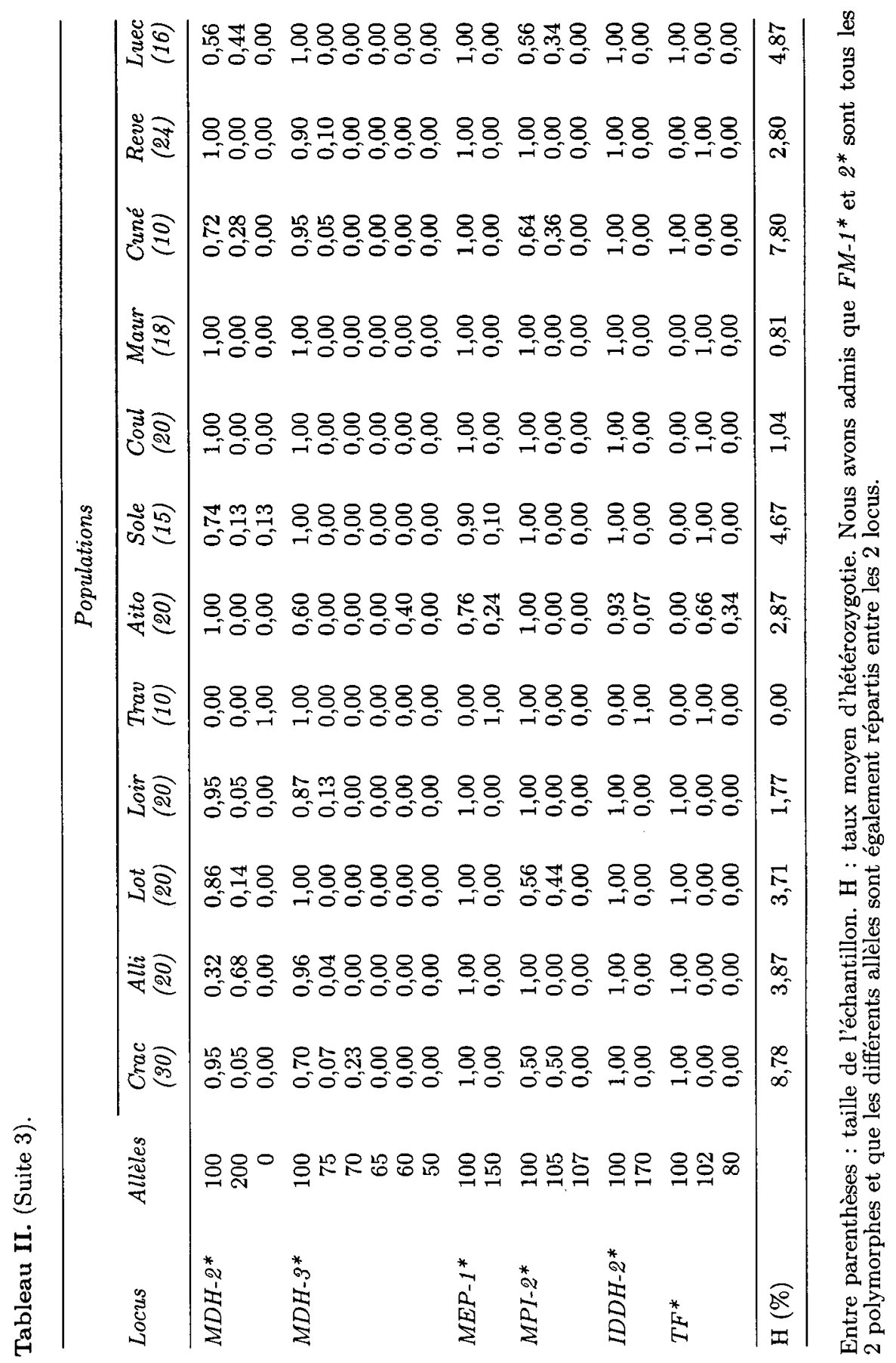




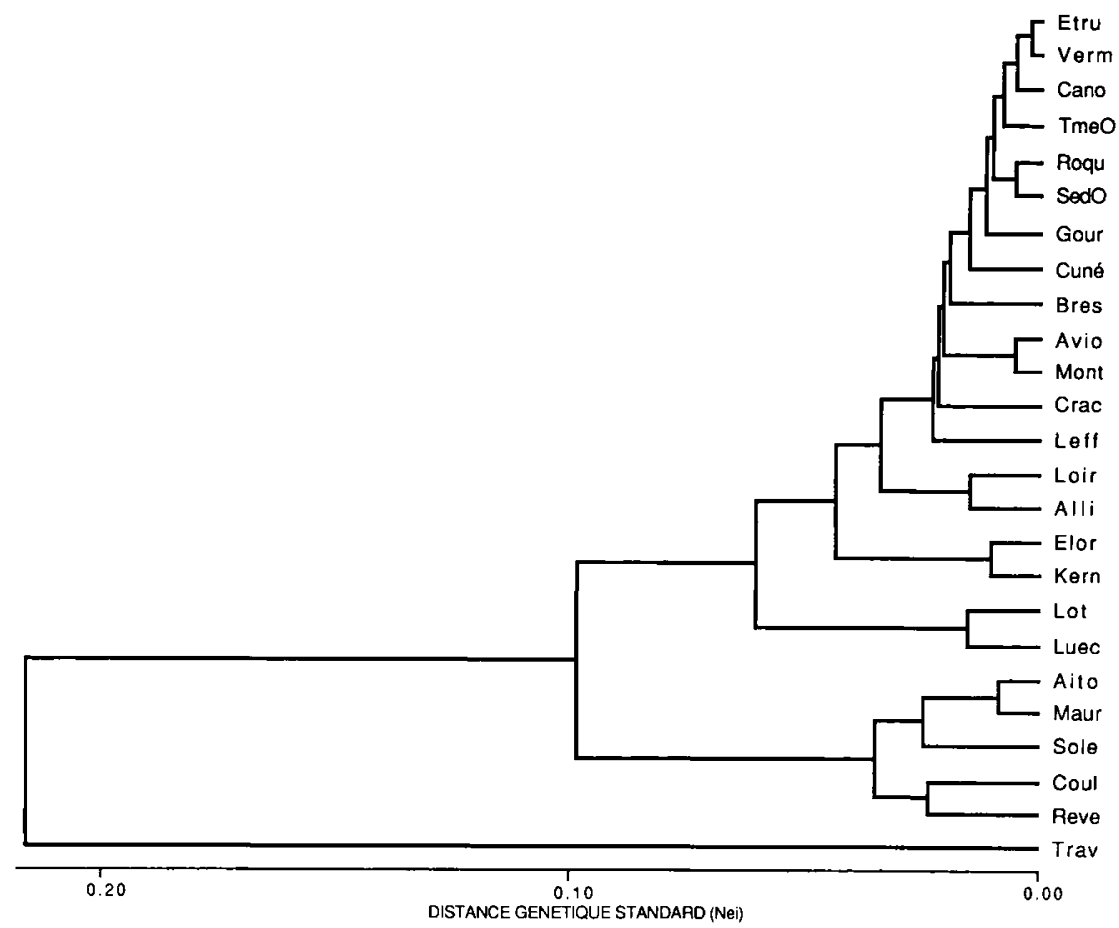

Fig 2. Dendrogramme obtenu par agglomération hiérarchique (UPGMA) avec les distances génétiques de Nei calculées à partir des fréquences alléliques observées aux 47 locus enzymatiques étudiés. Pour l'identification des populations, se reporter au tableau II.

\section{Variabilité des locus microsatellites}

Le criblage d'une banque génomique partielle composée de 863 clones à l'aide d'un mélange équimolaire d'oligonucléotides (GT)10 et (CT)10 a permis d'isoler 14 clones contenant des microsatellites (GT)n et 4 clones comportant des motifs (CT)n. La taille théorique moyenne des inserts clonés étant de $350 \mathrm{pb}$, on peut estimer le nombre total de paires de bases analysées à $350 \times 863=302000 \mathrm{bp}$. En supposant que la banque partielle criblée est représentative de l'ensemble du génome et que les séquences microsatellites sont régulièrement distribuées dans celui-ci, la distance moyenne entre 2 microsatellites (GT)n adjacents peut-être estimée par $302000 / 14=22000 \mathrm{bp}$. Une valeur de $75 \mathrm{~kb}$ est obtenue dans le cas des motifs (CT)n. A partir des séquences de 4 clones positifs, nous avons déterminé 4 couples d'amorces afin d'analyser le polymorphisme des locus microsatellites délimités par ces amorces. Trois des couples d'amorces permettent de visualiser la variabilité de locus uniques tandis que le quatrième couple révèle 2 locus simultanément. Cette duplication est peut-être à mettre en relation avec la tétraploïdisation qui s'est produite dans la famille des salmonidés au cours de son évolution (Ohno et al, 1969). Les 5 locus (nommés $\mathrm{m} 15, \mathrm{~m} 60, \mathrm{~m} 73, \mathrm{~m} 79-1$ et $\mathrm{m} 79-2$ ) présentent des variations 
inter- ou intrapopulation importantes : en dépit de la petite taille des échantillons examinés, les nombres d'allèles sont respectivement de $5,5,8,5$ et 2 pour les 5 locus. $\AA$ An locus donné, l'écart de tailles entre allèles de mobilités extrêmes ne sont jamais importants (20 bases dans le cas de m73). Les allèles observés dérivent les uns des autres par l'insertion ou la délétion d'un multiple de 2 bases correspondant à la taille d'un motif de base et presque tous les intermédiaires entre les allèles extrêmes ont été trouvés. En général, ces observations restent valables au niveau de chaque population. Des croisements de contrôle ont permis de vérifier que les ségrégations observées aux locus $\mathrm{m} 15, \mathrm{~m} 60$ et m73 sont mendéliennes (Estoup et al, sous presse). Le tableau III montre les fréquences observées à ces 5 locus dans 4 populations.

Le degré de différenciation le plus élevé apparaît à nouveau entre la population méditerranéenne d'une part, et les 2 souches domestiques et la population atlantique, d'autre part. Deux locus, m79-1 et 2, discriminent parfaitement la population de la Réverotte des 3 autres populations et des différences de fréquences alléliques importantes existent à 2 autres locus, m15 et m73. Seul le locus m60 présente le même allèle commun dans les 4 échantillons. Les taux moyens d'hétérozygotie sont relativement élevés (valeurs comprises entre 0,23 et 0,35 ) et légèrement supérieurs dans les populations atlantiques. Les valeurs de PIC (indice qui permet d'apprécier la valeur d'un locus pour la cartographie : Botstein et al, 1980) varient de 0 à 0,74 selon le locus et la population.

\section{DISCUSSION ET CONCLUSION}

Les résultats présentés dans cet article montrent l'existence, chez les populations naturelles de truite commune, d'une variabilité génétique importante et fortement structurée sur le plan géographique.

Le premier fait marquant est la différenciation, clairement mise en évidence par le polymorphisme enzymatique entre les populations méditerranéennes et atlantiques. Il est vraisemblable que ces 2 groupes possèdent chacun une aire de distribution plus vaste. Les populations analysées dans les îles britanniques (Ferguson and Fleming, 1983; Stephens and McAndrew, 1990) et en Scandinavie (Ryman, 1983) présentent des variations électrophorétiques impliquant très probablement les mêmes allèles que ceux qui sont observés dans les populations atlantiques françaises. Les populations des tributaires de l'Océan Atlantique, de la Mer du Nord et de la Mer Baltique auraient donc une origine monophylétique par rapport aux populations méditerranéennes.

Il est intéressant de noter que certaines populations d'Irlande et d'Écosse, mais aussi d'Islande (Hamilton et al, 1989) et des tributaires atlantique de l'Espagne (Hamilton et al, 1989; Garcia-Marin et al, 1991; Martinez et al, 1993) possèdent l'allèle $L D H-5^{*} 100$ à des fréquences très élevées. Il faut aussi remarquer que cet allèle est très commun chez la plupart des autres espèces de salmonidés et que, jusqu'à présent, $L D H-5^{*} g 0$ n'a été trouvé que chez la truite commune (Hamilton et al, 1989; Guyomard, résultats non publiés). $L D H-5^{*} 100$ et $L D H-5^{*} g 0$ peuvent donc être respectivement considérés comme la forme ancestrale et la forme dérivée. À partir de la distribution géographique de cet allèle, Hamilton et al (1989) ont proposé un scénario de colonisation post-glaciaire de la facade atlantique européenne par la truite commune. Selon ce scénario, les bassins hydrographiques 
Tableau III. Fréquences alléliques observées à 5 microsatellites dans 4 populations de truite commune. La nomenclature des allèles est donnée comme suit : code du microsatellite-taille de l'allèle en paires de bases. La taille des échantillons est de 10, sauf dans le cas de la Reverotte (24 individus).

\begin{tabular}{|c|c|c|c|c|c|}
\hline Locus $\mu$ Sat & Allèle & Gourmay & Cuneo & Bresle & Reverotte \\
\hline \multirow[t]{5}{*}{$\mu 60$} & $60-97$ & 0,00 & 0,00 & 0,05 & 0,02 \\
\hline & $60-101$ & 0,60 & 0,65 & 0,65 & 0,98 \\
\hline & $60-103$ & 0,05 & 0,00 & 0,00 & 0,00 \\
\hline & $60-105$ & 0,35 & 0,30 & 0,30 & 0,00 \\
\hline & $60-107$ & 0,00 & 0,05 & 0,00 & 0,00 \\
\hline$H^{*}$ & & 0,51 & 0,49 & 0,49 & 0,04 \\
\hline$P I C^{* *}$ & & 0,42 & 0,41 & 0,41 & 0,04 \\
\hline \multirow[t]{7}{*}{$\mu 73$} & $73-138$ & 0,00 & 0,00 & 0,00 & 0,04 \\
\hline & $73-140$ & 0,15 & 0,15 & 0,10 & 0,04 \\
\hline & 73-142 & 0,00 & 0,00 & 0,15 & 0,92 \\
\hline & $73-144$ & 0,75 & 0,30 & 0,10 & 0,00 \\
\hline & $73-146$ & 0,10 & 0,45 & 0,65 & 0,00 \\
\hline & $73-150$ & 0,00 & 0,05 & 0,00 & 0,00 \\
\hline & $73-158$ & 0,00 & 0,05 & 0,00 & 0,00 \\
\hline $\mathrm{H}$ & & 0,41 & 0,68 & 0,53 & 0,15 \\
\hline PIC & & 0,37 & 0,63 & 0,49 & 0,14 \\
\hline \multirow[t]{5}{*}{$\mu 15$} & $15-214$ & 0,00 & 0,05 & 0,00 & 0,00 \\
\hline & $15-218$ & 0,05 & 0,10 & 0,30 & 0,21 \\
\hline & $15-220$ & 0,20 & 0,10 & 0,25 & 0,79 \\
\hline & $15-222$ & 0,50 & 0,60 & 0,15 & 0,00 \\
\hline & $15-224$ & 0,25 & 0,15 & 0,30 & 0,00 \\
\hline $\mathbf{H}$ & & 0,65 & 0,60 & 0,74 & 0,33 \\
\hline PIC & & 0,59 & 0,57 & 0,69 & 0,27 \\
\hline \multirow[t]{4}{*}{$\mu 79-1$} & $79-1-126$ & 0,00 & 0,00 & 0,00 & 0,21 \\
\hline & $79-1-124$ & 0,00 & 0,00 & 0,00 & 0,33 \\
\hline & $79-1-122$ & 0,00 & 0,00 & 0,00 & 0,46 \\
\hline & $79-1-120$ & 1,00 & 1,00 & 1,00 & 0,00 \\
\hline $\mathrm{H}$ & & 0,00 & 0,00 & 0,00 & 0,64 \\
\hline PIC & & 0,00 & 0,00 & 0,00 & 0,57 \\
\hline \multirow[t]{2}{*}{$\mu 79-2$} & $79-2-116$ & 1,00 & 1,00 & 1,00 & 0,00 \\
\hline & $79-2-114$ & 0,00 & 0,00 & 0,00 & 1,00 \\
\hline $\mathrm{H}$ & & 0,00 & 0,00 & 0,00 & 0,00 \\
\hline PIC & & 0,00 & 0,00 & 0,00 & 0,00 \\
\hline $\mathrm{H}$ moyen & & 0,31 & 0,35 & 0,35 & 0,23 \\
\hline
\end{tabular}

* H : taux d'hétérozygotie; ** PIC : polymorphism information content.

devenus accessibles après le retrait des glaces auraient d'abord été réoccupés par une race «ancestrale» fixée pour l'allèle $L D H-5^{*} 100$, puis auraient subit une seconde colonisation par une forme moderne, caractérisée par $L D H-5^{*} 90$. Les mêmes auteurs suggèrent que l'allèle $L D H-5^{*} 90$, et par conséquent la race moderne, seraient apparus dans la mer baltique ou la mer du nord. L'allèle ancestral se serait maintenu 
jusqu'à nos jours dans certaines zones rendues temporairement ou définitivement inaccessibles à la race moderne. Certaines des hypothèses sur lesquelles s'appuie cette tentative de reconstitution paléogéographique sont, sans doute, vraies. Il est, en effet, très probable que la forme $L D H-5^{*} g 0$ soit dérivée de $L D H-5^{*} 100$ à la suite d'une mutation qui se serait produite exclusivement dans la zone Atlantique-Mer du Nord-Mer Baltique. Il peut également être admis, selon un principe de parcimonie d'hypothèses, que cette mutation se soit produite après la dernière glaciation. Par contre, il n'est pas possible, à partir des données disponibles, de déterminer la zone d'origine de cette mutation : toutes les populations des bassins de la Loire et de la Garonne que nous avons examinées sont naturellement fixées pour $L D H-5^{*} g 0$. Enfin, il paraît prématuré de parler de races géographiques à partir de différences alléliques à un seul locus. À ce sujet, il serait intéressant de vérifier s'il existe une corrélation entre les fréquences des allèles $F H-1,2 * 75, L D H-5^{*} 100$ et $S O D-1{ }^{*} 80$ dans les populations naturelles atlantiques non contaminées par le repeuplement.

Peu de données ont été, jusqu'à présent, publiées sur les populations méditerranéennes : 7 populations grecques avec 25 locus examinés (Karakousis et Triantaphyllidis, 1990) et une population espagnole du bassin de l'Ebre avec 50 locus analysés (Garcia-Marin et al, 1991). Une comparaison est possible uniquement avec la seconde étude qui comporte aussi l'analyse de populations atlantiques et domestiques. Il apparaît que la population de l'Ebre ne comporte pas un certain nombre d'allèles que nous n'avons trouvés que dans les populations d'origine atlantique et est presque fixée pour les allèles $L D H-5^{*} 100$ et $M P I-2^{*} 105$. Elle pourrait donc être génétiquement très peu différente des populations méditerranéennes décrites dans cet article. Par contre, l'étude de populations naturelles de la vallée du Pô montre qu'il existe au moins une seconde entité génétique dans la zone méditerranéenne (Giuffra, 1993).

L'analyse préliminaire du polymorphisme de 5 locus microsatellite conduit à des conclusions analogues puisqu'elle permet de distinguer très clairement la population méditerranéenne des 3 populations d'origine atlantique. La différenciation observée entre ces 3 dernières peut, par contraste, paraitre faible. Il faut cependant relativiser la portée de ce résultat dans la mesure où l'origine des 2 populations domestiques n'est pas connue et où la Bresle a déjà fait l'objet de repeuplements. La comparaison de ces données de polymorphisme de microsatellites avec celles qui ont été obtenues dans des populations humaines (Edwards et al, 1992; McInnis et al, 1993; Valdes et al, 1993) indique que la variabilité intrapopulation et le nombre d'allèles de fréquence supérieure à $1 \%$ par locus (dans cette étude, environ 100 allèles par locus ont été examinés si l'on regroupe les 4 populations) sont plus élevés chez l'homme que chez la truite. Par contre, la différenciation entre populations est beaucoup plus marquée chez celle-ci. La mise en évidence d'allèles diagnostiques à certains locus doit cependant être considérée avec précaution. D'une part, les échantillons analysés sont de petite taille et les études réalisées chez l'homme sur des nombres plus importants d'individus font apparaître de nombreux allèles à faible fréquence. D'autre part, compte tenu de la distribution de la taille des allèles entre et dans les populations et si les taux de mutation sont suffisamment élevés à ces locus, il est probable que des allèles identiques réapparaissent localement par «convergence» dans des populations bien différenciées. 
La dichotomie entre versants atlantique et méditerranéen révélée par l'électrophorèse de protéines ainsi que par nos données préliminaires sur les locus microsatellites a été retrouvée lors de l'étude de polymorphisme de séquence de la région de contrôle de réplication (D-loop) de l'ADN mitochondrial (Bernatchez et al, 1992). Aucun polymorphisme de séquence n'a été trouvé dans la zone atlantique. Par contre, 2 clones mitochondriaux méditerranéens distincts ont été mis en évidence, mais leur distribution ne paraît cependant pas géographiquement cohérente. La divergence moyenne (exprimée en nombre moyen de substitutions de nucléotides par site) entre le clone mitochondrial atlantique et les 2 clones méditerranéens s'établit à 1,1\% (Bernatchez et al, 1992). La distance génétique standard de Nei, estimée à partir des données d'électrophorèse enzymatique (cet article), est d'environ $10 \%$ entre populations méditerranéennes et atlantiques. Cette distance exprime le nombre moyen de différences apparentes (c'est-à-dire modifiant la charge de la protéine) de codons par locus entre 2 populations. D'après Nei (1987), si l'on admet que le nombre moyen d'acides aminés est de 400 par protéine, soit 1200 nucléotides, et que l'électrophorèse de protéine ne révèle que $8,3 \%$ de substitution de nucléotides, le pourcentage moyen de divergence nucléotidique par site entre les 2 sous-espèces peut être estimé à $(10 \% / 8,3 \%) / 1200=0,1 \%$ pour les locus protéiques. La région de l'ADNmt qui a été séquencée accumule donc les substitutions de nucléotides de façon beaucoup plus importante que les locus protéiques. Il est possible que cet avantage soit dû, comme chez certains vertébrés supérieurs (Vawter and Brown, 1986), à un taux d'évolution de l'ADNmt plus élevé que celui de gènes nucléaires «simple copie», bien que des travaux plus récents suggèrent que l'ADNmt des téléostéens évolue beaucoup plus lentement que celui des oiseaux ou des mammifères (Kocher et al, 1989; Martin and Palumbi, 1993). Une influence prépondérante de facteurs démographiques (sex-ratios déséquilibrés, taux de migration différents selon le sexe) ne peut être exclue (Birky et al, 1989). L'étude du polymorphisme de séquence n'est cependant pas plus discriminante ici que l'étude du polymorphisme enzymatique car le nombre de nucléotides séquencés de la D-loop (640, en moyenne) est nettement inférieur aux nucléotides «utiles» examinés par électrophorèse de protéines et dont le nombre peut être estimé à 4500 environ pour 47 locus (Nei, 1987, p 253).

La comparaison avec d'autres espèces de salmonidés montre que l'ampleur et la structure de la variabilité intraspécifique diffèrent notablement d'une espèce à l'autre (fig 3).

Ces différences doivent être interprétées avec précaution : d'une part, la définition des espèces, essentiellement basée sur des critères morphologiques, peut présenter un caractère arbitraire dans certains cas et, d'autre part, les analyses électrophorétiques sont plus ou moins approfondies selon les espèces. La figure 3 montre cependant que la truite commune, malgré un échantillonnage restreint à une faible partie de son domaine, se place parmi les espèces les plus polymorphes et les plus différenciées. Il convient aussi de remarquer que la truite commune et le saumon atlantique, bien qu'étant apparemment proches par leur biologie, leur phylogénie et leur paléogéographie récente, présentent des structures génétiques très différentes, même lorsque la comparaison est limitée à la partie commune de leur aire naturelle de répartition.

Le second fait important mis en évidence dans cet article est l'origine exclusivement atlantique des souches d'élevage, quelle que soit la localisation géographique de la pisciculture dont elles proviennent. L'origine strictement atlantique de ces souches 


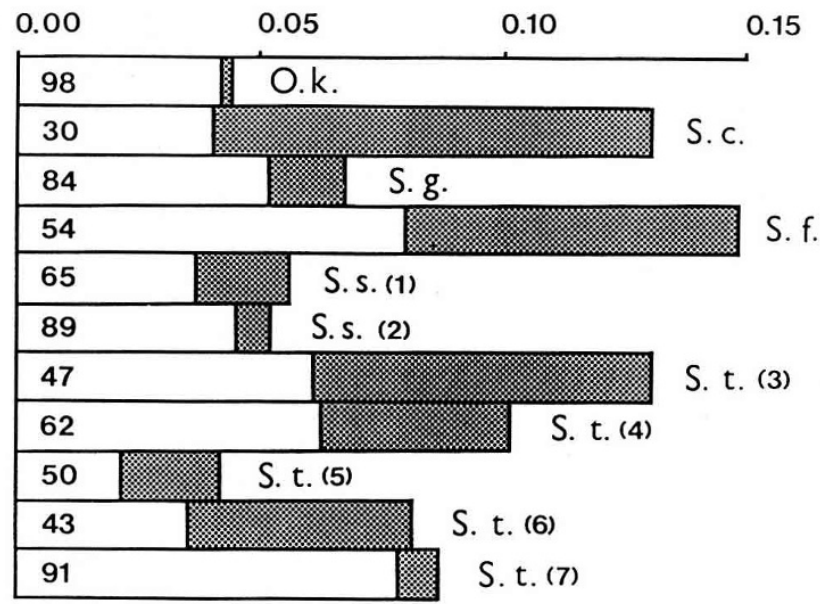

Fig 3. Comparaison des variabilités intra- $(H p)$ et inter-population $\left(D_{m}^{t}\right)$ moyennes estimées à partir des données électrophorétiques chez la truite commune et quelques espèces de salmonidés. Ok : Oncorhynchus keta, Okazaki, 1982; 54 populations, 22 locus. Sc : Oncorhynchus clarki, Loudenslager et Gall, $1980: 24$ populations, 35 locus. $\mathrm{S} \mathrm{g}$ : Oncorhynchus mykiss dans Ryman, 1983; 38 populations, 16 locus. S f Salvelinus fontinalis, Stoneking et al, 1981; 8 populations, 39 locus. Ss : Salmo salar, Guyomard, 1987 ; (1) : 10 populations européennes et canadiennes, 32 locus; (2) : 6 populations atlantiques européennes, 32 locus. S t Salmo trutta, cet article; (3) : 25 populations, 47 locus; (4) : populations atlantiques, 47 locus; $(5)$ : populations méditerranéennes, 47 locus; (6) populations corses, 47 locus; (7) : 7 souches de pisciculture, 47 locus. Les chiffres correspondent aux variabilités intrapopulation exprimées en pourcentage des variabilités totales. $\square \mathrm{Hp}$; 圈 $\mathrm{D}_{\mathrm{m}}^{\mathrm{t}}$.

apparaît également dans d'autres travaux (Garcia-Marin et al, 1991 ; Martinez et $a l, 1993)$ et dans les données de séquençage obtenues sur des sujets domestiques (Giuffra et al, 1993). Il est actuellement difficile de préciser la zone d'origine de ces souches qui sont probablement le produit de mélanges de populations et d'échanges entre piscicultures comme le suggèrent leurs hauts niveaux d'hétérozygotie et leurs faibles degrés de différenciation. Les raisons de cette origine exclusivement atlantique sont peut-être d'ordre purement historique (la pisciculture de la truite commune semble s'être d'abord développée sur le bassin du Rhin). Elles peuvent être d'ordre biologique; il faut en effet remarquer que les populations de type méditerranéen présentent des performances de survie et croissance en pisciculture particulièrement médiocres par rapport aux souches domestiques actuelles (Guyomard, résultats non publiés) et qu'il n'est pas prouvé que cette différence soit le résultat exclusif de la domestication.

L'étude des effets du repeuplement, à partir de la description des fréquences alléliques des locus les plus discriminants $\left(F B P-1^{*}, F H-1,2^{*}, L D H-5^{*}, M P I-2^{*}\right.$, $T F^{*}$ ), a conduit à mettre en évidence des phénomènes d'hybridation introgressive entre souches domestiques et populations autochtones dans les rivières méditerranéennes (Guyomard et Krieg, 1986; Barbat-Leterrier et al, 1989). L'ampleur de 
cette introgression est variable selon les sites (de 0 à $50 \%$ de gènes domestiques). Les déséquilibres de liaison observés dans certains cas (Barbat-Leterrier et al, 1989) sont difficiles à interpréter. Les croisements expérimentaux ne révèlent pas d'incompatibilité génétique entre les formes domestiques et méditerranéennes en première génération (Guyomard, résultats non publiés). Il ne peut pas toutefois être exclu que des mécanismes d'incompatibilité partielle se manifestent en seconde génération.

L'analyse détaillée des effets de l'introgression entre souches domestiques et populations naturelles, en particulier, la recherche de distorsions de ségrégations simples ou conjointes, la mise en évidence d'éventuelles contraintes sélectives régissant l'interpénétration de 2 complexes de gènes coadaptés distincts ainsi que l'évaluation des conséquences du repeuplement sur la biologie des populations naturelles nécessitent l'étude d'un nombre élevé de marqueurs génétiques sur des échantillons de grandes tailles. Contrairement aux locus enzymatiques, les locus microsatellites semblent bien adaptés à cet objectif. Leur densité dans le génome de la truite commune est relativement élevée (en moyenne, un microsatellite de type (CT)n tous les $25 \mathrm{~kb}$ environ) et du même ordre de grandeur que chez les vertébrés supérieurs (Stalling et al, 1991; Beckmann and Weber, 1992). Il semble possible d'obtenir rapidement, au moins pour des usages locaux, un nombre suffisant de locus diagnostiques. Leur polymorphisme peut être analysé à des stades très précoces du développement et sur de grands effectifs sans nécessairement sacrifier les individus échantillonnés.

La description de la structure génétique de la truite commune par électrophorèse de protéines a permis de mettre en évidence l'existence de 2 entités génétiques bien distinctes. La gestion actuelle de cette espèce, en particulier le mode de repeuplement basé sur le déversement d'un petit nombre de souches domestiques de même origine, ne respecte pas cette diversité. À partir des données déjà recueillies et des résultats de travaux portant sur la manipulation du niveau de plö̈die chez la truite commune (Quillet et al, 1991), il est possible de proposer, dans un premier temps à titre expérimental, une autre stratégie de gestion génétique des populations naturelles basée, d'une part, sur l'utilisation de populations domestiques triploïdes stériles permettant d'effectuer des repeuplements «non polluants» sur le plan génétique et, d'autre part, sur la recolonisation des milieux restaurés à partir d'individus sauvages prélevés dans des populations natives non contaminées par le repeuplement. La préservation de ces populations pourrait être garantie par la mise en place de "sanctuaires génétiques» qui seraient choisis et régulièrement contrôlés par analyse de polymorphisme moléculaire et bénéficieraient d'une réglementation particulière en matière de repeuplement et d'environnement. Ils constitueraient, en outre, des réservoirs de génotypes pour les programmes d'amélioration génétique susceptibles de se développer chez cette espèce. Cette stratégie de conservation des ressources génétiques serait relativement simple à appliquer et pourrait être transposée à d'autres espèces.

Enfin, il nous paraît utile de rappeler qu'il est illusoire de chercher à protéger la diversité génétique et à maintenir la valeur sélective des peuplements naturels si leur environnement se dégrade. Une gestion plus écosystémique, même ramenée à quelques actions simples, mais garantissant la qualité biologique et physico-chimique du milieu, devrait peu à peu se substituer aux politiques actuelles de gestions monospécifiques et souvent productivistes. 


\section{REMERCIEMENTS}

Les travaux présentés dans cet article ont été entrepris dans le cadre de l'AIP Prodige. Ils ont aussi bénéficié du concours financier du Bureau des ressources génétiques et de la CEE (projet AQ-2-493).

\section{RÉFÉRENCES}

Barbat-Leterrier A, Guyomard R, Krieg F (1989) Introgression between introduced domesticated strains and mediterranean native populations of brown trout (Salmo trutta L). Aquat Living Res 2, 215-223

Beckmann JS, Weber JL (1992) Survey of human and rat microsatellites. Genomics $12,627-631$

Bernatchez L, Guyomard R, Bonhomme F (1992) DNA sequence variation of the mitochondrial control region among geographically and morphologically remote European brown trout Salmo trutta populations. Mol Ecol 1, 161-173

Birky CW, Fuerst P, Maruyama T (1989) Organelle gene diversity under migration, mutation, and drift: equilibrium expectations, approach to equilibrium, effects of heteroplasmic cells, and comparison to nuclear genes. Genetics 121, 613-627

Botstein D, White RL, Skolnick M, Davis RW (1980) Construction of a genetic linkage map in man using restriction fragments length polymorphism. Am J Hum Genet 32, 314-331

Chevassus B, Krieg F, Guyomard R, Blanc JM, Quillet E (1992) The genetics of brown trout: twenty years of French research. Workshop on aquaculture of Arctic char and brown trout, 1991, Fludir, Iceland. Buvidinsi Icel Agr Sci 6, 109-124

Edwards A, Hammond HA, Jin L, Caskey CT, Chakraborty R (1992) Genetic variation at five trimeric and tetrameric tandem repeat loci in four human population groups. Genomics 12, 241-253

Estoup A (1992) Clonage de sondes microsatellites nucléaires locus spécifiques pour l'étude de la diversité génétique chez la truite commune (Salmo trutta L). Mémoire de DAA, ENSA de Rennes

Estoup A, Presa P, Krieg F, Vaiman D, Guyomard R (1993) $(\mathrm{CT})_{\mathbf{n}}$ and $(\mathrm{GT})_{\mathbf{n}}$ microsatellites: a new class of genetic markers for brown trout, Salmo trutta L. A paraître dans Heredity

Ferguson A, Fleming CC (1983) Evolutionary and taxonomic significance of protein variation in brown trout (Salmo trutta $\mathrm{L}$ ) and other salmonids. In: (D Rollinson, GS Oxford, eds), Protein polymorphism: adaptative and taxonomic significance. Academic press, London, 86-99

Garcia-Marin JL, Jorde PE, Ryman N, Utter F, Pla C (1991) Management implications of genetic differentiation between native and hatchery populations of brown trout (Salmo trutta) in Spain. Aquaculture 95, 235-249

Giuffra E (1993) Identificazione genetica e filogenia delle populazioni de trota comune, Salmo trutta L, del bacino del Po. Thèse de doctorat de recherches, Université de Turin

Giuffra E, Bernatchez L, Guyomard R (1993) Mitochondrial control and protein coding genes sequence variation among phenotypic forms of brown trout, Salmo trutta L, from Northern Italy. À paraître dans Mol Ecol 
Guyomard R (1987) Différentiation génétique des populations de saumon atlantique : revue et interprétation des données électrophorétiques et quantitatives. In: Restauration des rivières à saumons (Thibault M, Billard R, eds). Colloque BERGERAC, INRA, 298-308

Guyomard R, Krieg F (1983) Electrophoretic variation in 6 populations of brown trout (Salmo trutta L). Can J Genet Cytol 23, 403-413

Guyomard R, Krieg F (1986) Mise en évidence d'un flux génétique entre populations naturelles de truite fario et souche de repeuplement dans 2 rivières de Corse. Bull Fr Pêche Piscic 303, 134-140

Hamilton KE, Ferguson A, Taggart JB, Tomasson T, Walker A, Fahy E (1989) Post-glacial colonisation of brown trout, Salmo trutta L : Ldh-5 as phylogeographic marker locus. J Fish Biol

Karakousis Y, Triantaphyllidis C (1989) Genetic structure and differentiation among Greek brown trout (Salmo trutta L) populations. Heredity 64, 297-304

Kocher TD, Thomas WK, Meyer A, Edwards SV, Paäbo S, Villablanca FX, Wilson AC (1989) Dynamics of mitochondrial DNA evolution in animals: amplification and sequencing with conserved primers. Proc Natl Acad Sci USA 86, 6196-6200

Krieg F (1984) Recherche d'une différentiation génétique entre populations de Salmo trutta. Thèse de $3^{\mathbf{e}}$ cycle, Université Paris-Sud, Orsay

Krieg F, Guyomard R (1985) Population genetics of French brown trout (Salmo trutta L) : large geographical differentiation of wild populations and high similarity of domesticated stocks. Genet Sel Evol 17, 225-242

Krieg F, Quillet E, Chevassus B (1992) Brown trout, Salmo trutta L: a new species for intensive marine aquaculture. Aquacult Fish Manage 23, 557-566

Loudenslager EJ, Gall GAE (1980) Geographic patterns of protein variation and subspeciation in cutthroat trout (Salmo clarki). Syst Zool 28, 27-42

McInnis MG, Chakravarti A, Blaschak J et al (1993) A linkage map of human chromosome 21: 43 PCR markers at average intervals of $2.5 \mathrm{cM}$. Genomics 16, 562-571

Martin AP, Palumbi SR (1993) Body size, metabolic rate, generation time, and molecular clock. Proc Natl Acad Sci USA 90, 4087-4091

Martinez P, Arias J, Castro J, Sanchez L (1993) Differential stocking incidence in brown trout (Salmo trutta) populations from northwestern Spain. Aquaculture 114, 203-216

Nei M (1975) Molecular population genetics and evolution. North-Holland pub. co, Amsterdam

Nei M (1987) Molecular evolutionary genetics. Columbia University Press, New York

Ohno S, Muramoto J, Klein J, Atkin NB (1969) Diploid-tetraploid relationship in clupeoid and salmonid fish. In: Chromosome today, (Darlington CD, Lewis KR eds). Oliver and Boyd, Edinburgh,vol II, 139-147

Okazaki T (1982) Genetic study on population structure in chum salmon (Oncorhynchus keta). Bull Far Seas Fish Res Lab 19, 25-116

Quillet E, Foisil L, Chevassus B, Chourrout D, Liu FG (1991) Production of alltriploid and all-female brown trout for aquaculture. Aquat Living Res 4, 27-32

Ryman N (1983) Patterns of distribution of biochemical genetic variation in salmonids: differences between species. Aquaculture 33, 1-21 
Shaklee JB, Allendorf FW, Morizot DC, Whitt GS (1990) Gene nomenclature for protein coding loci in fish. Trans Amer Fish Soc 119, 2-15

Stallings RL, Ford AF, Nelson D, Torney DC, Hildebrand CE, Moyzis RK (1991) Evolution and distribution of (GT)n repetitive sequences in mammalian genomes. Genomics 10, 807-815

Stephen AB, Mc Andrew BJ (1990) Distribution of genetic variation in brown trout, Salmo trutta L. In: Scotland Aquat Fish Management 21, 47-66

Stoneking M, Wagner DJ, Hildebrand AC (1981) Genetic evidence suggesting subspecific differences between northern and southern populations of brook trout (Salvelinus fontinalis). Copeia 4, 810-819

Valdes AM, Slatkin M, Freimer NB (1993) Allele frequencies at microsatellite loci: The stepwise mutation model revisited. Genetics 133, 737-749

Vawter L, Brown WM (1986) Nuclear and mitochondrial DNA comparisons reveal extreme rate variation in the molecular clock. Sciences 234, 194-196 